\title{
Dispersion of the neutron resonance in cuprate superconductors
}

\author{
Andrey V. Chubukov, ${ }^{1,2}$ Boldizsár Jankó, ${ }^{1,3}$ and Oleg Tchernyshyov ${ }^{1,4}$ \\ ${ }^{1}$ Materials Sciences Division, Argonne National Laboratory, Argonne, Illinois 60439 \\ ${ }^{2}$ Department of Physics, University of Wisconsin, Madison, Wisconsin 53706 \\ ${ }^{3}$ Physics Department, University of Notre Dame, Notre Dame, Indiana 46556-5670 \\ ${ }^{4}$ School of Natural Sciences, Institute for Advanced Study, Princeton, New Jersey 08540
}

(October 22, 2018)

\begin{abstract}
We argue that recently measured downward dispersion of the neutron resonance peak in cuprate superconductors is naturally explained if the resonance is viewed as a spin-1 collective mode in a $\mathrm{d}$-wave superconductor. The reduction of the resonant frequency away from the antiferromagnetic wave vector is a direct consequence of the momentum dependence of the d-wave superconducting gap. When the magnetic correlation length becomes large, the dispersion should become magnonlike, i.e., curve upwards from $(\pi, \pi)$.
\end{abstract}

PACS numbers: 71.10.Ca, 74.20.Fg, 74.25.-q

Superconductivity and antiferromagnetism are two major phases of the high- $T_{c}$ cuprates. When viewed separately, the corresponding ground states appear to be quite conventional: the parent compounds of high $T_{c}$ cuprates (such as $\mathrm{La}_{2} \mathrm{CuO}_{4}$ 迎) are exemplary Heisenberg antiferromagnets, while overdoped cuprates in many respects resemble BCS-type d-wave superconductors [2]. However, full understanding of the interplay between the two phenomena is sorely lacking. Although most experts agree that antiferromagnetism is the ultimate cause of high- $T_{c}$ superconductivity, the intermediate steps are not yet clear [3].

Perhaps the strongest experimental indication of the interplay between antiferromagnetism and superconductivity in cuprates is the discovery of strong inelastic neutron scattering deep in the superconducting (SC) phase of materials with the highest $T_{c}$. The most intense scat-

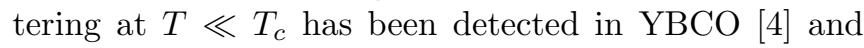
Bi2212 [5] near the antiferromagnetic (AF) wave vector $\mathbf{q}=\mathbf{Q}=(\pi, \pi)$ and energy $\Omega_{\mathbf{Q}} \approx 40 \mathrm{meV}$. Experiments conducted with polarized neutrons [6] indicate that the resonant scattering is due to electron spins.

It is tempting to interpret the resonance as a magnon in a disordered Néel state, which becomes more visible in a superconductor. However, a magnon frequency

$$
\Omega_{\mathbf{q}}^{2}=c^{2}\left(\xi^{-2}+|\mathbf{q}-\mathbf{Q}|^{2}\right)=\Omega_{\mathbf{Q}}^{2}+c^{2}|\mathbf{q}-\mathbf{Q}|^{2},
$$

where $c$ is a spin-wave velocity, clearly increases away from the $\mathrm{AF}$ wave vector $\mathbf{Q}=(\pi, \pi)$ [7]. Meanwhile, recent experiments on YBCO [8,9] have revealed the opposite trend: the resonance energy decreases away from $(\pi, \pi)$ [see Fig. 11(a)]. In a disordered antiferromagnet, this can only be the case if spin response in the normal state is incommensurate 110]. However, the data suggest [9] that, unlike in 214 materials [11], the normal-state spin response in YBCO is commensurate.

On second thought, the best superconductors of the cuprate family differ significantly from their parent AF compounds. They have a large, Luttinger-like Fermi surface in the normal state, and fermionic quasiparticles in the SC state, as shown by angle-resolved photoemission spectroscopy (ARPES) [12]. Several authors have demonstrated that in this situation, an attractive exchange force in the $\mathrm{d}$-wave superconducting state binds an electron and a hole into a pair with total spin 1 [13 16]. This bound state is seen as a resonance in a spin response. The gross features of the neutron resonance, such as a non-monotonic variation of $\Omega_{\mathbf{Q}}$ with doping and persistence of the resonance in the pseudogap phase can be understood if the resonance peak is viewed as a collective mode 14 16.

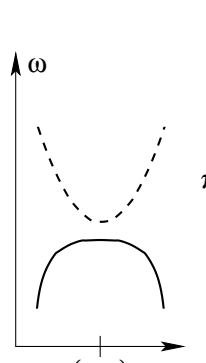

(a)

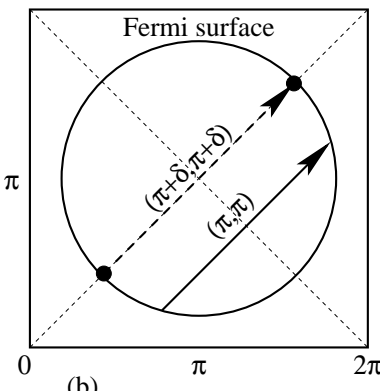

(b)

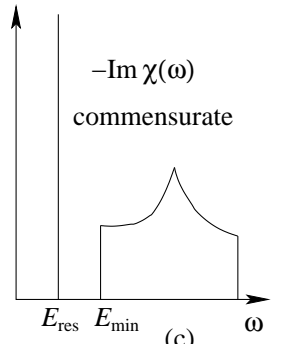

(c)
FIG. 1. (a) A sketch of the resonance dispersion: experimental (solid line), a massive magnon in the disordered Néel state (dashed line). (b) Approximate shape of the Fermi surface in Bi2212. Filled dots are nodal points, where the fermions are gapless. The continuum of two-particle excitations at the corresponding wave vector $\mathbf{q}_{0}=(\pi+\delta, \pi+\delta)$ (dashed arrow) starts at zero frequency. For lattice momentum $\mathbf{Q}=(\pi, \pi)$ (solid arrow) the continuum starts at a finite energy. (c) spin susceptibility at the wave vector $(\pi, \pi)$.

Formally, a collective mode has the dispersion given by Eq. (11). Its frequency is proportional to the static part of the inverse spin susceptibility $\left(\xi^{-2}+|\mathbf{q}-\mathbf{Q}|^{2}\right)^{1 / 2}[15]$. However, this time the velocity $c$ is not constant but depends rather strongly on the wave vector $\mathbf{q}$. This dependence can be understood by noting that the bound state necessarily resides below the two-particle continuum [Fig. 1 (c)]. For $\mathbf{q}$ along a zone diagonal, the continuum starts at $E_{\min }(\mathbf{q})=|\Delta(\mathbf{k})|+|\Delta(\mathbf{k}+\mathbf{q})|$, where 
both momenta $\mathbf{k}$ and $\mathbf{k}+\mathbf{q}$ are at the Fermi surface, Fig. 1(b). As $\mathbf{q}$ moves away from $(\pi, \pi), \mathbf{k}$ and $\mathbf{k}+\mathbf{q}$ shift along the Fermi surface towards the nodal points and the bound state inside the gap is pushed to lower energies. For $\mathbf{q}=\mathbf{q}_{0}$ connecting the nodal points, the two-particle spectrum is gapless, $E_{\min }\left(\mathbf{q}_{0}\right)=0$, so that the energy of the resonance must vanish (along with its strength). Obviously then, $\Omega_{\mathbf{q}}$ should decrease away from $(\pi, \pi)$, at least near $\mathbf{q}_{0}$.

The above qualitative argument is quite robust and is based on two premises only: (i) the Fermi surface contains hot spots (i.e., points connected by the $\mathrm{AF}$ wave vector $\mathbf{Q}$ ) and (ii) the superconducting gap has the $d_{x^{2}-y^{2}}$ symmetry. A more quantitative description of the resonance requires further assumptions. Most calculations of a spin response in a d-wave superconductor start with a model of free fermions on a square lattice, add an exchange interaction and employ the randomphase approximation (RPA) to compute spin susceptibility [13, 14, 16]. The downward dispersion of the resonance can then be obtained under favorable circumstances [16]. Unfortunately, such calculations must be done numerically providing somewhat limited insight. Here we present an alternative, analytical approach to the problem. It is based on the observation that the behavior of a collective mode is determined largely by low-energy fermion degrees of freedom and is therefore insensitive to the physics at high energies.

Our point of departure is a macroscopic spin-fermion model that describes low-energy fermionic quasiparticles (with a Fermi surface inferred from ARPES) interacting with collective spin fluctuations. The model is described by the effective action 17

$$
\begin{aligned}
S= & -\int_{0}^{\beta} d \tau \int_{0}^{\beta} d \tau^{\prime} \sum_{\mathbf{k}, \sigma} c_{\mathbf{k} \sigma}^{\dagger}(\tau) G_{0}^{-1}\left(\mathbf{k},\left(\tau-\tau^{\prime}\right)\right) c_{\mathbf{k} \sigma}\left(\tau^{\prime}\right) \\
& +\frac{1}{2} \int_{0}^{\beta} d \tau \int_{0}^{\beta} d \tau^{\prime} \sum_{\mathbf{q}} \chi_{0}^{-1}(\mathbf{q}, \tau) \mathbf{S}_{\mathbf{q}}(\tau) \cdot \mathbf{S}_{-\mathbf{q}}\left(\tau^{\prime}\right) \\
& +g \int_{0}^{\beta} d \tau \sum_{\mathbf{q}} \mathbf{s}_{\mathbf{q}}(\tau) \cdot \mathbf{S}_{-\mathbf{q}}(\tau)
\end{aligned}
$$

where $G_{0}^{-1}(\mathbf{k}, \tau)=\frac{\partial}{\partial \tau}-\mathbf{v}_{\mathbf{k}} \cdot\left(\mathbf{k}-\mathbf{k}_{F}\right)$ is the bare fermionic propagator, and $\chi_{0}^{-1}(\mathbf{q}, \tau)$ is the bare spin susceptibility. This model is viewed as a low-energy version of a lattice, Hubbard-type model, obtained by integrating out highenergy degrees of freedom. Accordingly, it has a cut-off $\Lambda<E_{F}$ and the bare $\chi_{0}(\mathbf{q}, \tau)$ is determined by fermions with energies exceeding $\Lambda$. If, as we assume, nothing special happens at high frequencies, $\chi_{0}(\mathbf{q}, \Omega)$, the Fourier transform of $\chi_{0}(\mathbf{q}, \tau)$ will have an Ornstein-Zernike form: $\chi_{0}(\mathbf{q}, \Omega)=\chi_{0} /\left(\xi^{-2}+(\mathbf{q}-\mathbf{Q})^{2}-\Omega^{2} / v_{s}^{2}\right)$.

Previous studies of Eq. (2) focused on renormalization of the fermionic dispersion by spin-fermion interaction, and on the form of the full $\chi(\mathbf{Q}, \Omega)[15]$. Here we consider the dynamical susceptibility at $\mathbf{q} \neq \mathbf{Q}$. To avoid unnecessary complications, we restrict calculations to momenta along the zone diagonal, $\mathbf{q}=(q, q)$. We also neglect strong coupling effects, assuming for simplicity that superconductivity is described by a d-wave version of the BCS theory. We have verified that strong-coupling effects (which modify fermionic propagator at low energies) result in quantitative, but not qualitative changes.

The full dynamical susceptibility $\chi(\mathbf{q}, \Omega)$ differs from $\chi_{0}(\mathbf{q}, \Omega)$ due to a bosonic self-energy $\Pi(\mathbf{q}, \Omega)$ :

$$
\chi(\mathbf{q}, \Omega)=\frac{\chi_{0}}{\xi^{-2}+(\mathbf{q}-\mathbf{Q})^{2}-\Omega^{2} / v_{s}^{2}-\Pi(\mathbf{q}, \Omega)} .
$$

The static part of $\Pi(\mathbf{q}, \Omega)$ (the contribution of the lowenergy fermions to the inverse correlation length $1 / \xi)$ is small and, in fact, vanishes for linearized fermion dispersion. On the other hand, the frequency-dependent part of $\Pi(\mathbf{q}, \Omega)$ is substantially nonzero in the normal state. This is related to the fact that, for a Fermi surface with hot spots (as in YBCO and Bi2212) a low-frequency spin excitation with momentum $\mathbf{q} \approx \mathbf{Q}$ can decay into two fermions at the Fermi surface (Fig. 1). This gives rise to a universal relaxational term in $\Pi(\mathbf{q}, \Omega)$ :

$$
\Pi(\mathbf{q}, \Omega) \approx i \operatorname{Im} \Pi(\mathbf{q}, \Omega)=i|\Omega| \gamma_{\mathbf{q}}
$$

At small frequencies, $\Pi(\mathbf{q}, \Omega) \propto|\Omega|$ is much larger than the bare term $\mathcal{O}\left(\Omega^{2}\right)$ in the susceptibility. It thus fully determines spin dynamics at low energies. The prefactor $\gamma_{\mathbf{q}}$ is obtained by calculating the imaginary part of the particle-hole bubble. In two dimensions, $\gamma_{\mathbf{q}}=$ $2 \bar{g} /\left(\pi v_{x} v_{y}\right)$, where $\bar{g}=g^{2} \chi_{0}$ is the effective spin-fermion coupling, and $\mathbf{v}=\left(v_{x}, v_{y}\right)$ is a (q-dependent) Fermi velocity at the hot spot $\mathbf{k}_{\mathrm{hs}}(\mathbf{q})$, whose components are defined as $\epsilon_{\mathbf{k}}=v_{x} \tilde{k}_{x}+v_{y} \tilde{k}_{y}, \epsilon_{\mathbf{k}+\mathbf{q}}=-v_{x} \tilde{k}_{x}+v_{y} \tilde{k}_{y}$, where $\tilde{\mathbf{k}}=\mathbf{k}-\mathbf{k}_{\mathrm{hs}}(\mathbf{q})$ [15]. Ordinarily, the $\mathbf{q}$ dependence is weak and can be neglected. However, for the wave vector $\mathbf{q}_{0}$ connecting the nodes, the velocities at $\mathbf{k}_{\mathrm{hs}}$ and $\mathbf{k}_{\mathrm{hs}}+\mathbf{q}_{0}$ are antiparallel, therefore $v_{y}=0$ and $\gamma_{\mathbf{q}}$ diverges. We have verified that $\Pi\left(\mathbf{q}_{0}, \Omega\right)$ is of order $\sqrt{\Omega}$.

Eqs. (3) and (4) imply that in the normal state

$$
\operatorname{Im} \chi(\mathbf{q}, \Omega) \propto \frac{x}{\left(1+\tilde{q}^{2} \xi^{2}\right)^{2}+x^{2}}
$$

where $x=\Omega / \gamma \xi^{2}$, and $\tilde{\mathbf{q}}=\mathbf{q}-\mathbf{Q}$. We see that spin response is (a) incoherent: no sharp peak in $\chi^{\prime \prime}(\mathbf{q}, \Omega)$ as a function of frequency $\Omega$; (b) commensurate: $\chi^{\prime \prime}(\mathbf{q}, \Omega)$ is peaked at $\mathbf{q}=\mathbf{Q}$ at a fixed $\Omega$. Both results are in agreement with the data 11.

In a d-wave superconducting state, the bosonic selfenergy is modified thanks to the opening of a superconducting gap. It is now given by

$\Pi(\mathbf{q}, \Omega)=\frac{i \gamma_{\mathbf{q}}}{2} \int\left(1-\frac{\omega(\omega+\Omega)-\Delta_{\mathbf{q}}^{2}}{\sqrt{\left[\omega^{2}-\Delta_{\mathbf{q}}^{2}\right]\left[(\omega+\Omega)^{2}-\Delta_{\mathbf{q}}^{2}\right]}}\right) d \omega$. 
Here $\Delta_{\mathbf{q}}$ is the fermion gap at one of the hot spots connected by $\mathbf{q}$, i.e., $\Delta_{\mathbf{q}}=\Delta(\mathbf{k})$, such that both $\mathbf{k}$ and $\mathbf{k}+\mathbf{q}$ are at the Fermi surface. By virtue of the $d_{x^{2}-y^{2}}$ symmetry, $\Delta(\mathbf{k})=-\Delta(\mathbf{k}+\mathbf{q})$, a condition we used in deriving Eq. (6).

In the presence of a superconducting gap with the dwave symmetry, $\operatorname{Im} \Pi(\mathbf{q}, \Omega)$ vanishes discontinuously for $\Omega<2\left|\Delta_{\mathbf{q}}\right|$. By virtue of the Kramers-Kronig relation, this discontinuity generates a nonzero $\operatorname{Re} \Pi(\mathbf{q}, \Omega)$ that is quadratic in $\Omega$ at low frequencies:

$$
\Pi(\mathbf{q}, \Omega) \sim \gamma_{\mathbf{q}} \Omega^{2} / \Delta_{\mathbf{q}} \quad \text { as } \Omega \rightarrow 0 .
$$

It is also essential that, for any $\mathbf{q}, \Pi(\mathbf{q}, 0)=0$ : the opening of the d-wave gap does not change the magnetic correlation length. This result is not entirely surprising: spinmediated d-wave pairing involves only fermions from opposite sublattices and thus does not affect the correlation of spin within the same sublattice.

Substitution of Eq. (7) into (3) yields

$$
\chi(\mathbf{q}, \Omega) \propto \frac{c_{\mathbf{q}}^{2}}{c_{\mathbf{q}}^{2}\left(\xi^{-2}+\tilde{q}^{2}\right)-\Omega^{2}}, \quad c_{\mathbf{q}}^{2}=\frac{\Delta_{\mathbf{q}}}{\gamma_{\mathbf{q}} \xi^{2}} .
$$

We see that in a $d$-wave superconductor the low energy spin excitations are propagating, gapped magnonlike modes with the dispersion

$$
\Omega_{\mathbf{q}}^{2}=c_{\mathbf{q}}^{2}\left(\xi^{-2}+|\mathbf{q}-\mathbf{Q}|^{2}\right)
$$

Eqs. (8)-(9) are meaningful only if $c_{\mathbf{q}} \xi^{-1}<\left|\Delta_{\mathbf{q}}\right|$. Otherwise the use of a quadratic form for $\Pi(\mathbf{q}, \Omega)$ is not justified. Strictly speaking, near $\mathbf{q}=\mathbf{Q}, c_{\mathbf{q}} \xi^{-1}<\Delta_{\mathbf{q}}$ only at sufficiently strong coupling, when fermion self-energy cannot be neglected. On the other hand, a quadratic frequency dependence of $\operatorname{Re} \Pi(\mathbf{q}, \Omega)$ at low $\Omega$ is merely a consequence of a vanishing $\operatorname{Im}(\mathbf{q}, \Omega)$ for $\Omega<2\left|\Delta_{\mathbf{q}}\right|$, where at strong coupling $\Delta_{\mathbf{q}}$ should be understood as the measured gap (i.e., a frequency where the spectral function has a $\delta$-functional peak). We have explicitly verified that inclusion of strong coupling corrections into (6) only changes the overall factor in (7).

To verify that our analytical approach [based on a linearized fermion dispersion around $\mathbf{k}_{\mathrm{hs}}(\mathbf{q})$ ] captures the essential features of the spin susceptibility, we present in Fig. 2 numerical results for the bare particle-hole susceptibility $\tilde{\chi}_{0}(\mathbf{q}, \Omega)$ and its RPA counterpart $\tilde{\chi}(\mathbf{q}, \Omega)$ $\tilde{\chi}(\mathbf{q}, \Omega)=\tilde{\chi}_{0}(\mathbf{q}, \Omega) /\left[1-J \tilde{\chi}_{0}(\mathbf{q}, \Omega)\right]$. Both are calculated for a $d_{x^{2}-y^{2}}$ BCS superconductor with a tight-binding dispersion $\epsilon_{k}$ inferred from ARPES data [16]. We expect that, at low enough $\Omega, \Pi(\mathbf{q}, \Omega)$ matches $\tilde{\chi}_{0}(\mathbf{q}, \Omega)$, modulo a regular function of $q$. Similarly, $\chi(\mathbf{q}, \Omega)$ should agree, at low frequencies, with $\tilde{\chi}(\mathbf{q}, \Omega)$.

In Fig. 2, we see that $\operatorname{Im} \tilde{\chi}_{0}(\mathbf{q}, \Omega)=0$ for $\Omega<2\left|\Delta_{\mathbf{q}}\right|$ and has a finite jump at $2 \Delta_{\mathbf{q}}$, while $\operatorname{Re} \tilde{\chi}_{0}(\mathbf{q}, \Omega)$ is quadratic in $\Omega$ at low frequencies, in agreement with what we obtained for $\Pi(\mathbf{q}, \Omega)$. The peaks in $\operatorname{Re} \tilde{\chi}_{0}(\mathbf{q}, \Omega)$ at $2 \Delta_{\mathbf{q}}$ are logarithmic singularities associated with the discontinuity of $\operatorname{Im} \tilde{\chi}_{0}\left(2 \Delta_{\mathbf{q}}\right)$. They are softened because we used complex frequencies with a small imaginary part. These singularities are indeed present in $\Pi(\mathbf{q}, \Omega)$. A strong peak in the RPA susceptibility $\tilde{\chi}(\mathbf{q}, \Omega)$ is the resonance located at $1=J \operatorname{Re} \tilde{\chi}_{0}(\mathbf{q}, \Omega)$. This expression corresponds to $\Pi(\mathbf{q}, \Omega)=\xi^{-2}+(\mathbf{q}-\mathbf{Q})^{2}$ in our analytical approach. We have also verified that the static susceptibility $\tilde{\chi}_{0}(\mathbf{q}, 0)$ does not change between normal and superconducting states (i.e., $\xi$ is not renormalized). High-frequency features in Fig 2 -not not captured by our long-wavelength approximation - are lattice effects sensitive to the details of the fermion dispersion $\epsilon_{\mathbf{k}}$ far from the Fermi surface.

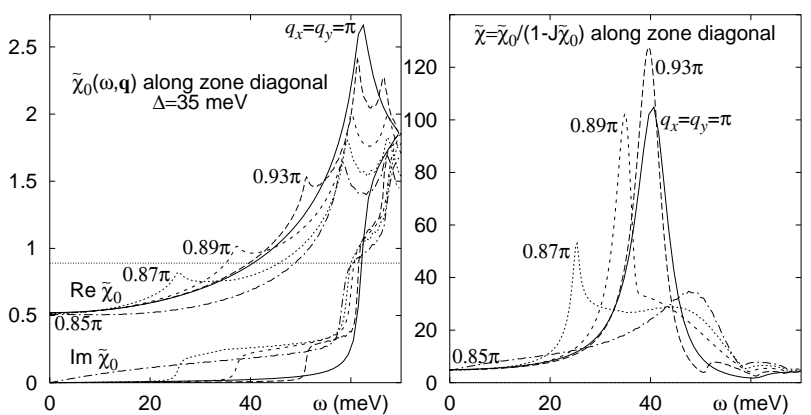

FIG. 2. Left: real and imaginary parts of the numerically evaluated particle-hole bubble in a $d$-wave superconductor, $\tilde{\chi}_{0}(\mathbf{q}, \omega)$, at various $\mathbf{q}=(q, q)$, using a tight-binding fermion dispersion inferred from ARPES. The low-frequency features of $\tilde{\chi}_{0}(\mathbf{q}, \omega)$ are in agreement with our analytical results. Right: imaginary part of the corresponding full dynamical susceptibility, $\operatorname{Im} \chi(\Omega, \mathbf{q})$. Observe that the resonance moves to lower frequencies when $\mathbf{q} \neq \mathbf{Q}=(\pi, \pi)$.

We now analyze Eq. (9). If $\Delta_{\mathbf{q}}$ and $\gamma_{\mathbf{q}}$ were independent of momentum, $c_{\mathbf{q}}$ would be constant and $\Omega_{\mathbf{q}}$ would be a converntional magnon-like dispersion (11). In this situation, the spin resonance would remain commensurate and exist only at $\Omega>\Omega_{\mathbf{Q}}=c_{\mathbf{Q}} / \xi$. However, in a d-wave superconductor, $\Delta_{\mathbf{q}}$ decreases and $\gamma_{\mathbf{q}}$ increases when $\mathbf{q}$ deviates from $\mathbf{Q}$. This effect accounts for the downward shift of $\Omega_{\mathbf{q}}$. Furthermore, as $c_{\mathbf{q}}$ vanishes at $\mathbf{q} \rightarrow \mathbf{q}_{0}$, the resonance frequency $\Omega_{\mathbf{q}} \rightarrow 0$ regardless of the spin correlation length $\xi$. Quite generally, near $\mathbf{q}_{0}$, $\operatorname{Im} \chi(\mathbf{q}, \Omega)$ should have a peak at a frequency $\Omega_{\mathbf{q}}<\Omega_{\mathbf{Q}}$.

The behavior near $\mathbf{q}=\mathbf{Q}$ is more complicated and depends on the values of $\xi$ and $\mathbf{q}_{0}$. We can roughly approximate the momentum dependence of $c_{\mathbf{q}}$ as

$$
c_{\mathbf{q}}^{2} \approx 1-\left(\tilde{q} / \tilde{q}_{0}\right)^{2}, \quad \tilde{q}=|\mathbf{q}-\mathbf{Q}| .
$$

While the specific functional form we adopt here for $c_{\mathbf{q}}$ is unimportant for capturing the qualitative behavior of the neutron resonance dispersion, the form adopted in Eq. (10) is appropriate for a magnetically mediated d-wave superconductivity in which $\Delta_{\mathbf{q}}$ is largest at $\mathbf{q}=\mathbf{Q}$ [17].

Upon substituting this form into Eq. (9), we find 


$$
\frac{\Omega_{\mathbf{q}}}{\Omega_{\mathbf{Q}}}=1-\left(\frac{\tilde{q}}{\tilde{q}_{0}}\right)^{2}\left(1-\left(\tilde{q}_{0} \xi\right)^{2}\right)-\left(\frac{\tilde{q}}{\tilde{q}_{0}}\right)^{4}\left(\tilde{q}_{0} \xi\right)^{2}
$$

When $\tilde{q}_{0} \xi$ is small, the dispersion is negative for all momenta. Also, the residue of the peak in Eq. (8) scales as $c_{\mathbf{q}}$, i.e., it decreases and eventually vanishes as $\mathbf{q}$ approaches $\mathbf{q}_{0}$.

In Fig. 3 we plot $\Omega_{\mathbf{q}}$ (11) and the intensity of the peak for $\tilde{q}_{0} \xi=2$. This value is consistent with optimally doped Bi2212 where $\left|\mathbf{q}_{0}\right| \approx 0.3 \pi / a$ [18]) while $\xi=a-2 a$ [19]. $\Omega_{\mathbf{q}}$ in (11) is rather flat near $\mathbf{Q}$ and rapidly drops away from $\mathbf{Q}$. The residue of the peak at the downturn is already much smaller than its value at $\mathbf{Q}$. Both these features are consistent with the data [9].
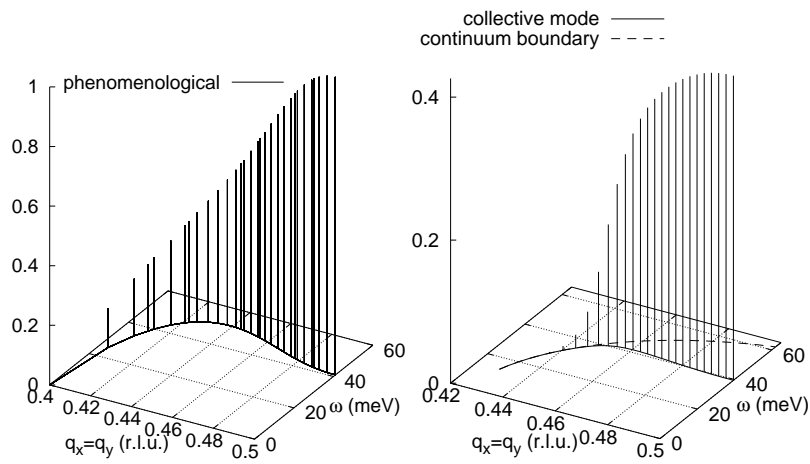

FIG. 3. Location and intensity of the resonance peak obtained from Fig. 目 (left figure) and from Eqs. (8) and (11) (right figure).

For comparison, we also present in Fig. 3 the dispersion and intensity of the collective mode obtained in the RPA calculation, Fig. 2. It exhibits qualitatively similar behavior which we interpret as evidence that the dispersion of the peak is insensitive to the details of the fermion dispersion at high energies. Note also that our estimate of the wave vector at which the energy and intensity of the neutron peak vanishes, $\mathbf{q}_{0} \approx(0.8 \pi, 0.8 \pi)$, is roughly consistent with the corresponding value found in neutron scattering, $\mathbf{q}=(\pi, 0.8 \pi)$. Recall that, to simplify the analysis, we have only considered momenta along zone diagonal.

Finally, we comment on the limit $\tilde{q}_{0} \xi \rightarrow \infty$ relevant to the underdoped side. In this limit, $\Omega_{\mathbf{q}}$ first increases with $\tilde{q}$ and drops only very near $\mathbf{q}=\mathbf{q}_{\mathbf{0}}$. This implies that the resonance continuously evolves into the magnon of the AF state. It would be of interest to verify experimentally whether such evolution takes place.

To summarize, we have demonstrated that the experimentally observed downturn of the resonant frequency [9] away from $(\pi, \pi)$, accompanied by a rapid decrease of the peak intensity, occurs rather naturally when the resonance peak is interpreted as a collective spin excitation in a d-wave superconductor. The unusual dispersion of the peak is related to a variation of the superconducting gap along the Fermi surface. We have argued that the resonant frequency vanishes at a certain $\mathbf{q}=\mathbf{q}_{0}$ that connects nodal points at the Fermi surface. Close to the commensurate wave vector $\mathbf{Q}=(\pi, \pi)$, the dispersion depends on the spin correlation length: If the latter is small, $\left|\mathbf{q}_{0}-\mathbf{Q}\right| \xi \ll 1$, the resonance frequency $\Omega_{\mathbf{q}}$ decreases with $|\mathbf{q}-\mathbf{Q}|$. Conversely, when $\left|\mathbf{q}_{0}-\mathbf{Q}\right| \xi \gg 1, \Omega_{\mathbf{q}}$ increases away from $\mathbf{Q}$, reminiscent of a magnon dispersion in a disordered antiferromagnet.

We acknowledge useful conversations with Ar. Abanov, J.C. Campuzano, P. Dai, M.R. Norman and S. Sachdev. The research was supported in part by the NSF Grant No. DMR-9979749, the US DOE Grant No. W31-109-ENG-38 and DE-FG02-90ER40542. A.Ch. and O.T. thank the Materials Science Division of Argonne National Laboratory for hospitality during their stay at Argonne.

[1] T. E. Mason in Handbook on the Physics and Chemistry of Rare Earths, edited by K. A. Gschneider Jr. et al.; cond-mat/9812297.

[2] J. C. Campuzano et al., Phys. Rev. B 53, R 14737 (1996).

[3] Proceedings of M2S-HTC-VI 2000, edited by C. W. Chu et al., Physica $\mathrm{C}$ (in press).

[4] J. Rossat-Mignod et al, Physica C 185, 86 (1991); H. F. Fong et al., Phys. Rev. Lett. 75, 316 (1995); P. Dai et al., Science 284, 1344 (1999).

[5] H. F. Fong et al., Nature 398, 588 (1999).

[6] H. A. Mook et al., Phys. Rev. Lett. 70, 3490 (1993).

[7] S. Sachdev, Quantum Phase Transitions, Cambridge University Press (Cambridge, 1999).

[8] M. Arai et al., Phys. Rev. Lett. 83, 608 (1999).

[9] P. Bourges et al., Science 288, 1234 (2000).

[10] M. Vojta, C. Buragohain, and S. Sachdev, Phys. Rev. B 61, 15152 (2000).

[11] See, for example, G. Aeppli et al., Science 278, 1432 (1997) and references therein.

[12] See, for example, M. R. Norman et al., Phys. Rev. Lett. 79, 3506 (1997); Z. X. Shen et al., Science 280, 259 (1998); P. V. Bogdanov et al., ibid. 84, 2581 (2000); H. M. Fretwell et al., Phys. Rev. Lett. 84, 4449, (2000).

[13] C. Stemmann et al., Phys. Rev. B 50, 4075 (1994); D. Z. Liu, Y. Zha and K. Levin, Phys. Rev. Lett. 75, 4130 (1995); I. Mazin and V. Yakovenko, ibid. 75, 4134 (1995); A. Millis and H. Monien, Phys. Rev. B 54, 16172 (1996); N. Bulut and D. Scalapino, Phys. Rev. B 53, 5149 (1996); D. K. Morr and D. Pines, Phys. Rev. Lett. 81, 1086 (1998); S. Sachdev and M. Vojta, Physica B 280, 333 (2000); Y.-J. Kao et al., Phys. Rev. B 61, R11898 (2000); F. Onufrieva and P. Pfeuty, cond-mat/9903097, O. Tchernyshyov et al., cond-mat/0009072;

[14] J. Brinckmann and P.A. Lee, Phys. Rev. Lett. 82, 2915 (1999).

[15] Ar. Abanov and A. Chubukov, Phys. Rev. Lett. 83, 1652 
(1999).

[16] M. R. Norman, Phys. Rev. B 61, 14751 (2000); cond-mat/0010298.

[17] Ar. Abanov et al., cond-mat/0010403.

[18] T. Valla et al., Science, 285, 2210 (1999).

[19] see e.g., D. J. Scalapino, Phys. Rep. 250, 329 (1995); D. Pines, Z. Phys. B 103, 129 (1997) and references therein. 